\title{
Pathway-Affecting Single Nucleotide Polymorphisms (SNPs) in RPS6KA1 and MBIP Genes are Associated with Breast Cancer Risk
}

\author{
Ghadah Shareefi ${ }^{1}$, Alaa Nabil Turkistani ${ }^{1}$, Ahmed Alsayyah ${ }^{2}$, Haitham Kussaibi ${ }^{2}$, \\ Maha Abdel Hadi ${ }^{3}$, Khaled R Alkharsah ${ }^{1 *}$
}

\begin{abstract}
Background: Genetic mutations and polymorphisms play an important role in the transformation of primary cells to malignant cells as it may lead to disturbance of vital pathways regulating cell cycle, DNA damage repair, and apoptosis. In this study, we genotyped single nucleotide polymorphisms (SNPs) which were predicted to affect certain pathways and to increase the risk of breast cancer. Methods: The study included 81 Saudi breast cancer patients and 100 matching healthy controls from the Eastern Province in Saudi Arabia. The following SNPs (rs3168891, rs2899849, rs2230394, rs2229714) were then genotyped by TaqMan genotyping assay and the allele and genotype distribution was compared. Results: The minor allele frequency of the following SNPs (rs3168891, rs2899849, rs2230394, rs2229714) was $\mathrm{T}=0.17, \mathrm{~A}=0.28, \mathrm{~A}=0.22$, and $\mathrm{G}=0.16$ respectively. The $\mathrm{G}$ allele of the $\mathrm{SNP}$ rs 3168891 was significantly associated with increased breast cancer risk $(\mathrm{P}=0.00001)$ while the $\mathrm{T}$ allele of the same locus was associated with reduced risk of breast cancer in both heterozygous and homozygous states. The T allele of SNP rs 2229714 which is located in the RPS6KA1 gene was also significantly associated with the increased risk of breast cancer. However, the $r s 2899849$ SNP located in the Integrin beta-1 (ITGB1) gene was not associated with the increased risk of breast cancer in our study population. Haplotype analysis revealed the presence of three risk haplotypes that increases the risk of breast cancer (TGGT, TGTA, GATA). Conclusion: We showed that three, previously untested, SNPs are associated with increased risk of breast cancer in our population. This may be added to the list of factors involved in breast cancer risk assessment studies. The benefit and the utility of the in-silico prediction of disease risk factors and their genetic association had been demonstrated in this study, yet the predicted risk alleles have to be tested in clinical studies.
\end{abstract}

Keywords: Breast cancer- SNP- pathway- MAPK- Saudi Arabia

Asian Pac J Cancer Prev, 21 (7), 2163-2168

\section{Introduction}

The latest Global Cancer statistics indicates that breast cancer was expected to account for a quarter of cancer cases amongst women in 2018 (Bray et al., 2018). Genetic predisposition plays a crucial role in breast cancer development. Up to $10 \%$ of breast cancer cases can be attributed to hereditary factors comprising personal or family history of breast or ovarian cancer and inherited gene mutations such as $B R C A 1$ and $B R C A 2$ among others (Eccles et al., 2013; Bray et al., 2018). Genetic mutations and polymorphisms in the human genome could also be linked to the risk of sporadic breast cancer especially in those genes related to cell development and differentiation. One of the early attempts to investigate the correlation between $p 21$ and $b c l 2 S N P S$ with susceptibility to breast cancer showed that $p 21$ was more commonly expressed in breast cancer patients and $b c l 2$ polymorphism was likely to be associated with breast cancer risk (Johnson et al., 2008). Interestingly, hundreds of SNPs have been associated with breast cancer risk so far (Turnbull et al., 2010; Eccles et al., 2013).

Genome-wide association studies (GWAS) facilitated the identification of a large number of breast cancer susceptibility alleles with a much lower size effect (Easton et al., 2007; Stacey et al., 2007; Turnbull et al., 2010; Michailidou et al., 2013). Although GWAS studies have shed light on the discovery and validation of novel diseasecausing gene mutations and polymorphisms, it remains a challenge to prove the link between the identified risk allele and the biological mechanism of its involvement in the disease process (Stacey et al., 2007).

${ }^{1}$ Department of Microbiology, College of Medicine, Imam Abdulrahman Bin Faisal University (IAU), Dammam, Saudi Arabia. ${ }^{2}$ Department of Pathology, College of Medicine, Imam Abdulrahman Bin Faisal University (IAU), Dammam, Saudi Arabia. ${ }^{3}$ Breast Division, Department of Surgery, College of Medicine, Imam Abdulrahman Bin Faisal University (IAU), Dammam, Saudi Arabia.*For Correspondence: kalkharsah@iau.edu.sa 
Since the transformation of breast epithelial cells to malignant cells involves the disturbance of vital pathways regulating cell cycle, DNA damage repair and apoptosis, Lee et al. designed software to identify the causal SNP from GWAS and their candidate affected pathway in an attempt to link the causal SNPs to a hypothetical mechanism of the disease (Lee et al., 2014). They identified several candidate SNPs among which four are with very strong putative biological mechanisms located in RPS6KA1, ITGB1, and MBIP genes, which modify the growth hormone signaling, PTEN pathway, and the mitogen-activated protein kinase (MAPK) pathways respectively (Lee et al., 2014).

A previous study from Saudi Arabia showed that breast cancer was the ninth prominent cause of death in women (Memish et al., 2014). In contrast to the global decrease in Breast cancer mortality, the incidence of breast cancer has shown a steady incline in Saudi Arabia, with the Eastern province reporting the highest incidence in the country (El Bcheraoui et al., 2015). Previous studies have found an association of breast cancer with multiple variants and SNPs among the Saudi population. A mutation variant within the 3'-untranslated region (3'-UTR) of the $B R C A 1$ gene and the $V E G F-2578 A A$ genotype were found to be associated with increased risk of breast cancer in among Saudi females (Al Balawi et al., 2018; Mir et al., 2018b). While other allele variants such as the progesterone receptor gene mutations and the microRNA-423 T allele were associated with advanced stage and metastasis of breast cancer (Mir et al., 2018a; Albalawi et al., 2020).

We sought in this study to identify the correlation between SNPs (rs3168891, rs2899849, rs2230394, rs2229714) and breast cancer risk among the Saudi population. These SNPs have been previously identified in GWAS in the following genes RPS6KA1 (rs2229714), ITGB1 (rs2230394), and MBIP (rs3168891 and $r s 2899849$ ) (Lee et al., 2014). These SNPs are expected to play a role in pathways that are vital for cell survival and development.

\section{Materials and Methods}

\section{Patients and Methods \\ Patients and controls}

Saudi female patients diagnosed with breast cancer or with a history of breast cancer regardless of their treatment regimen were recruited for the study. Patients were attending the breast cancer unit at King Fahd Hospital of the University (KFHU) in Alkhobar for interventional treatment or follow up. The targeted control group was Saudi females over the age of 40 years with no history of breast cancer or familial history of breast cancer up to second-grade relatives.

Informed consent was obtained from all patients and control individuals. The ethical approval for the study was obtained from the Institutional Review Board at Imam Abdulrahman Bin Faisal University (number IRB-UGS2017-01-092).

\section{DNA extraction}

Blood samples were collected in 3ml EDTA vacutainer tubes. DNA was then extracted using DNA extraction kit from Qiagen according to the instructions (Qiagen, Hilden, Germany). The quality and quantity of extracted DNA were measured by NanoDrop 2000 spectrophotometer. DNA was stored at $-20^{\circ} \mathrm{C}$ till the time of analysis.

\section{SNP genotyping}

Four SNPs (rs3168891, rs2899849, rs2230394, rs2229714) were selected from the publication which described and used the prediction software ICSNPathway to predict the pathway associated SNPs that may affect the risk of breast cancer from genome-wide association studies (Lee et al., 2014). The targeted SNPs were genotyped using TaqMan SNP genotyping Assay (Life Technologies, CA, USA). The real time-polymerase chain reaction (RT-PCR) was performed in $25 \mathrm{ul}$ reactions and was run on the thermocycler 7500Fast from applied Biosystems (Thermofisher Scientific, USA) according to the manufacturer's instruction. Figure 1 shows an example of the SNP genotyping results. The final allelic discrimination of each SNP was performed by the genotyping software associated with thermocycler 7500 version 2.0.6 from ABI (Figure 1). Each allele will be detected with a probe bound to certain fluorescence dye. The presence of the alleles will be reported based on the amplified signal detection of the corresponding dye and then separated the on a plot representing on one axis the first allele and on the other axis the second allele (Figure 1). In case of heterozygous genotype, both alleles will be detected (Figure 1).

\section{Statistical analysis}

SPSS software version 24 was used to calculate the Chi-square. Logistic regression was used to estimate the association between SNPs and the risk of breast cancer at a confidence interval of 95\%. All studied SNPs were found to be consistent with Hardy-Weinberg equilibrium. Linkage disequilibrium (LD) and the risk of the possible haplotypes were analyzed using the HaploView software version 4.2. A P value of less than 0.05 was considered significant.

\section{Results}

\section{Study population}

Eighty-one breast cancer female patients and one hundred control female individuals were recruited to the study. The age range for the patients was 26-79 years (median 50) at the time of diagnosis and for the control was 40-78 years (median 51). Patients' diagnostic parameters are summarized in Table 1.

Association between the studied SNPs and Breast cancer

There was a statistically significant association between SNP rs3168891 and the risk of breast cancer. The G allele at this location was significantly associated with increased risk of breast cancer $(\mathrm{OR}=4.1, \mathrm{P}=0.00001)$ (Table 2) while the $T$ allele of the same locus was associated with reduced risk of breast cancer in both heterozygous and homozygous states (Table 2). Figure 1 shows a representative result for the rs3168891 genotyping with 
Table 1. Clinical Data of Breast Cancer Patients in the Study

\begin{tabular}{|c|c|c|}
\hline & $\mathrm{N}=81 *$ & $\%$ \\
\hline Age & 77 & \\
\hline less than 40 & 16 & 20.8 \\
\hline more than 40 & 61 & 79.2 \\
\hline Tumor stage & 67 & \\
\hline Early (O, I and II) & 51 & 76.1 \\
\hline Late (III and IV) & 16 & 23.9 \\
\hline Tumor grade & 67 & \\
\hline Grade I & 8 & 11.9 \\
\hline Grade II & 30 & 44.8 \\
\hline Grade III & 29 & 43.3 \\
\hline Estrogen receptor expression (ER) & 70 & \\
\hline positive & 48 & 68.6 \\
\hline negative & 22 & 31.4 \\
\hline Progesterone Receptor expression & 70 & \\
\hline positive & 38 & 54.3 \\
\hline negative & 32 & 45.7 \\
\hline Her2/neu proteins expression & 68 & \\
\hline positive & 30 & 44.1 \\
\hline negative & 38 & 55.9 \\
\hline Distant Metastasis & 69 & \\
\hline positive & 4 & 5.8 \\
\hline negative & 65 & 94.2 \\
\hline
\end{tabular}

TaqMan Assay. The T allele of the rs2899849 SNP was associated with risk of breast cancer only in the homozygous state (Table 2). No association was found between $r s 2230394$ which is located in the IBTG1 gene and the risk of breast cancer (Table 2). The $\mathrm{T}$ allele of
SNP rs2229714 which is located in the RPS6KA1 gene was also significantly associated with increased risk of breast cancer (Table 2).

\section{Association of studied SNPS with patient clinical parameters}

There was no significant statistical association between any of the studied SNPs and other biometric parameters such as age at diagnosis, stage or grade of cancer, or the expression of hormone receptors.

\section{Haplotype analysis}

Analysis of the haplotypes in the study revealed that three haplotypes are associated with increased breast cancer risk in our study (Figure 2). The haplotypes are TGGT (p-value 0.001), TGTA (p-value 0.238), and GATA (p-value 0.0028) (Figure 2). These genotypes were generated from the studied SNPs in the following order rs2229714, rs2230394, rs3168891, and rs2899849. There was linkage disequilibrium between SNPs rs3168891 and rs2899849 (LOD score $>2$ ), which are located adjacent to each other in the $M B I P 1$ gene but not with any of the other SNPs.

\section{Discussion}

Breast cancer is emerging as a common female cancer in Saudi Arabia, with a higher incidence reported in the Eastern Province. Multiple studies investigated the association of specific SNPs with breast cancer risk. A significant association was found between breast cancer and the GG genotype of the rs1799950 SNP which is located in the BRCAl gene (Merdad et al., 2015). The TP53 gene, a tumor suppressor gene, is one of the most commonly downregulated genes among cancers in humans, including breast cancer (Al-Qasem
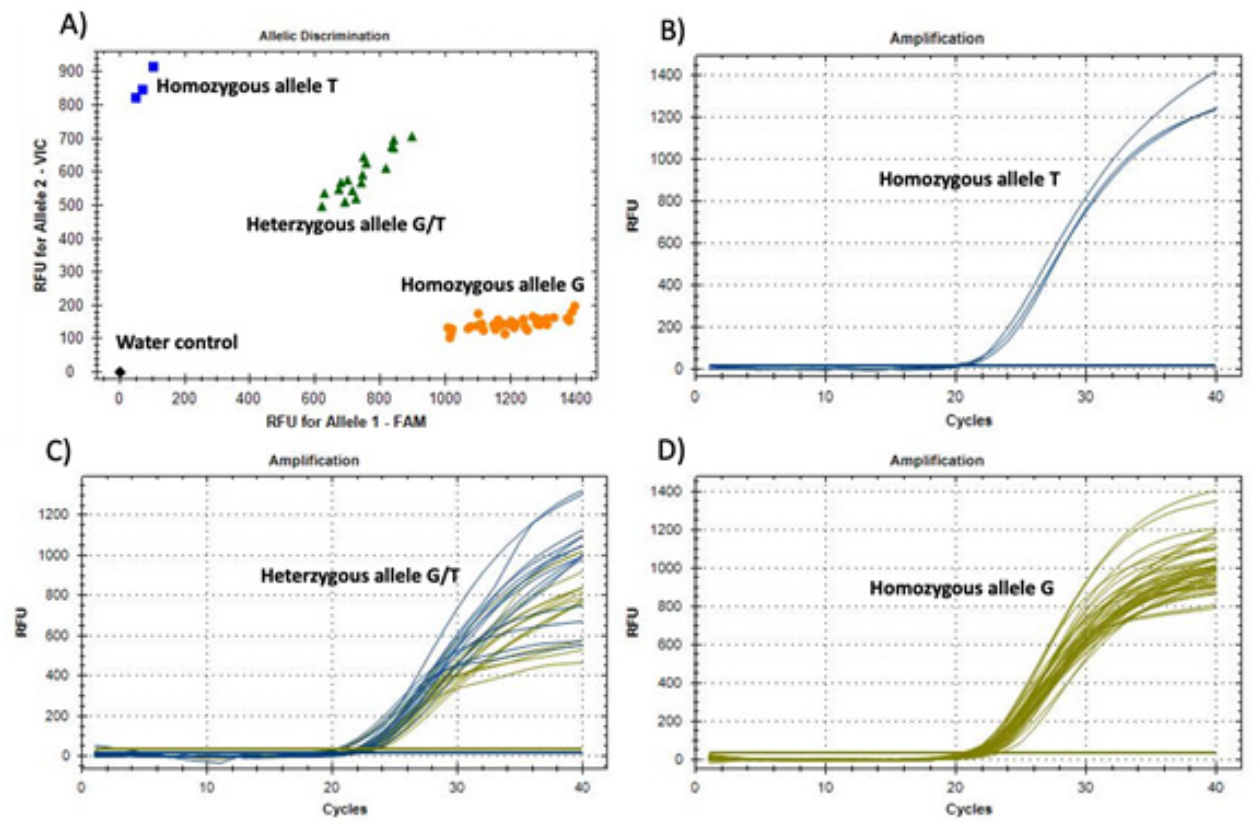

Figure 1. A representative result for the SNP rs3168891 (G/T) genotyping using TaqMan SNP assay. A) Shows the allelic discrimination plot. B) Shows amplification curves for the homozygous allele T. C) Shows amplification curves for the heterozygous allele ( $\mathrm{G}$ green/T blue). D) Shows amplification curves for the homozygous allele G 
Table 2. Allele and Genotype Analysis of the Studied SNPs Revealed an Association between the Pathway Affecting SNPs in MBIP and RPS6KA1 and Risk of Breast Cancer

\begin{tabular}{|c|c|c|c|c|c|}
\hline & Allele/Genotype & Patients $\mathrm{N}=81$ & Controls $\mathrm{N}=100$ & $P$-value & OR $(95 \% \mathrm{CI})$ \\
\hline \multicolumn{6}{|c|}{ MAP3K12 binding inhibitory protein $(M B I P)$} \\
\hline \multirow[t]{5}{*}{ rs3168891 } & $\mathrm{G}$ & 150 & 150 & 0.00001 & $4.1(2.2-8.4)$ \\
\hline & $\mathrm{T}$ & 12 & 50 & & \\
\hline & GG & 69 & 54 & 0.00001 & $4.9(2.4-10.4)$ \\
\hline & TG & 12 & 42 & 0.0001 & $0.2(0.1-0.5)$ \\
\hline & TT & 0 & 4 & $0.04 *$ & undefined \\
\hline \multirow[t]{5}{*}{ rs2899849 } & $\mathrm{T}$ & 133 & 145 & 0.042 & $1.7(1.1-2.9)$ \\
\hline & A & 29 & 55 & & \\
\hline & TT & 55 & 52 & 0.03 & $2.0(1.1-3.6)$ \\
\hline & TA & 23 & 41 & 0.1 & $0.6(0.3-1.1)$ \\
\hline & AA & 3 & 7 & 0.36 & $2.0(0.5-9.6)$ \\
\hline \multicolumn{6}{|c|}{ Integrin subunit beta $1(I T G B 1)$} \\
\hline \multirow[t]{5}{*}{ rs2230394 } & G & 137 & 157 & 0.18 & $1.5(0.9-2.6)$ \\
\hline & $\mathrm{A}$ & 25 & 43 & & \\
\hline & GG & 56 & 60 & 0.26 & $1.5(0.8-2.8)$ \\
\hline & GA & 25 & 37 & 0.47 & $0.8(0.4-1.4)$ \\
\hline & $\mathrm{AA}$ & 0 & 3 & 0.16 & undefined \\
\hline \multicolumn{6}{|c|}{ Ribosomal protein S6 kinase A1 (RPS6KA1) } \\
\hline \multirow[t]{5}{*}{ rs2229714 } & $\mathrm{T}$ & 147 & 158 & 0.002 & $2.6(1.4-5.0)$ \\
\hline & $\mathrm{G}$ & 15 & 42 & & \\
\hline & $\mathrm{TT}$ & 67 & 62 & 0.002 & $3.0(1.5-6.1)$ \\
\hline & TG & 13 & 34 & 0.01 & $0.4(0.2-0.8)$ \\
\hline & GG & 1 & 4 & 0.3 & $0.3(0.01-2.5)$ \\
\hline
\end{tabular}

*Mid-P exact test

et al., 2011). A study from Riyad revealed a significant association between R72P located in exon 4 of the TP53 gene and the early start of breast cancer among the Saudi population (Al-Qasem et al., 2012). In particular, the RP genotype was found to be protecting against breast cancer (Al-Qasem et al., 2012). However, all studies focus on genes involved in DNA repair machinery, while the transformation of breast epithelial cells to malignant cells may involve the disturbance of vital pathways regulating cell cycle and apoptosis. Lee et al. designed software called "ICSNPathway" to identify the causal SNP from GWAS and their candidate affected pathway in an attempt to link the causal SNPs to a hypothetical mechanism of the disease biology (Lee et al., 2014). They identified several candidate SNPs among which four are with very strong putative biological mechanisms located

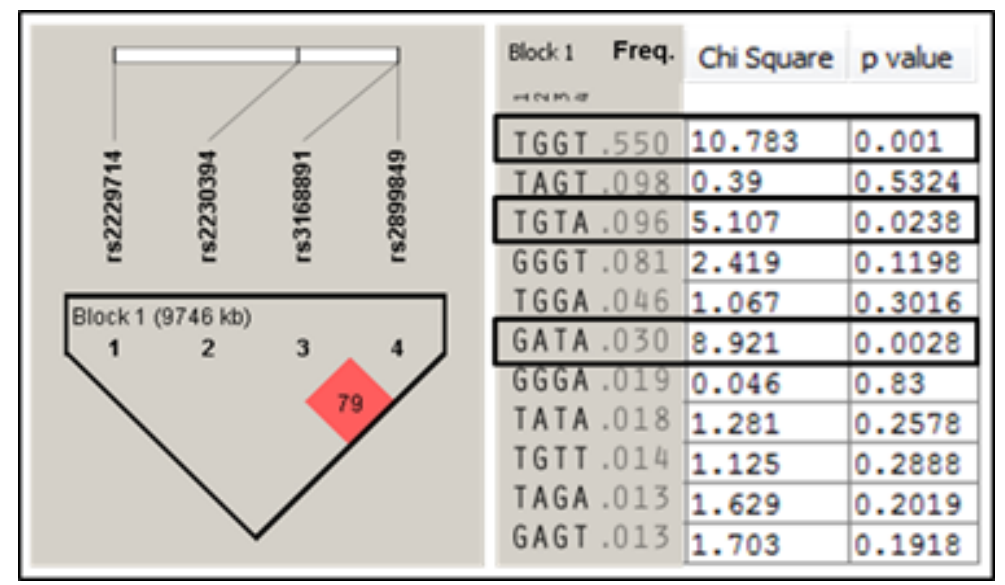

Figure 2. Haplotype Analysis of the Studied SNPs. The left panel shows linkage disequilibrium between SNPs 3 (rs3168891) and 4 (rs2899849) (LOD score > 2), which are located adjacent to each other in the MBIP1 gene but not with any of the other SNPs. The middle panel shows the frequency of the haplotypes in the study population. The right panel shows the statistical association between these haplotypes and breast cancer. The bolded boxed shows the haplotypes significantly associated with breast cancer. 
in RPS6KA1, ITGB1, and MBIP genes, which modify the growth hormone signaling, PTEN pathway, and the mitogen-activated protein kinase (MAPK) pathways respectively (Lee et al., 2014).

This current study focuses on the correlation of the following SNPs; rs3168891, rs2899849, rs2230394, and $r s 2229714$ predicted by the ICSNPathway software with breast cancer risk in Saudi patients. It is important to note that none of these SNPs were previously described to be associated with the risk of cancer.

The SNPs rs 3168891 and $r s 2899849$ are located adjacent to each other in the MBIP gene, which affects the MAPK pathway. Both SNPs were found to be associated with breast cancer in our study on both allele and genotype levels. The association was stronger with $r s 3168891$. The association with $r s 2899849$ seems to be influenced by rs3168891 because they are in linkage disequilibrium (LD) (Figure 2). MAPK pathway regulates many vital cellular functions such as cell differentiation, cellular growth, and apoptosis and the disruption of this pathway plays a role in tumorigenesis (Aguirre-Ghiso et al., 2003; Giltnane and Balko, 2014).

The SNP rs 2229714 was also significantly associated with breast cancer in our study. This SNP is located in the ribosomal protein S6 kinase $A 1$ gene (RPS6KA1), which encodes a serine/threonine kinase. The RPS6KA1 is one of the S6 kinase family of proteins ( $\mathrm{Li}$ et al., 2012). The ribosomal S6 kinase family of proteins is the downstream targets of the MAPK pathway effectors and in their turn phosphorylate several regulatory proteins involved in cell growth and differentiation (Casalvieri et al., 2017). Currently, several members of this family are considered promising targets for antiviral therapy (Casalvieri et al., 2017). Furthermore, these proteins are implicated in growth hormone signaling which plays an important role in breast normal physiology through driving the differentiation of ductal epithelium and normal proliferation secretory functions of lobular epithelial cells and disruption of these functions would promote tumor formation (Xu et al., 2011).

The rs2230394 was not associated with breast cancer in our study population. This SNP is located in the gene encoding integrin subunit beta 1 (ITGB1) and was expected to affect the PTEN and has 04360 pathways. Both pathways are expected to play an important role in breast cancer (DeGraffenried et al., 2004; Luu et al., 2013). However, this SNP seems not to play an important role in the genetic predisposition of breast cancer in our population. Haplotype analysis showed that the most frequent haplotype in the study population indeed consists of the risk alleles of the three SNPs associated with breast cancer in the study (Figure 2).

In conclusion, breast cancer is emerging as an alarming health burden in Saudi Arabia. Extensive studies should be directed towards identifying the factors that predispose to its increased incidence and discovering the pertinent risk factors. We showed that three previously untested SNPs are associated with breast cancer in our population. This may be added to the long list of factors involved in breast cancer risk assessment studies. The benefit of the utility of the software in the prediction of disease risk factors and the genetic association had been demonstrated in this study, yet, on a cautionary note, the predicted risk alleles have to be tested in clinical studies.

\section{Acknowledgements}

The authors are grateful for the patients and control individuals for their participation in the study. This Project was funded by Deanship for Scientific Research at Imam Abdulrahman Bin Faisal University [Project number 2016-575-Med].

\section{Declarations}

Funding: This project was funded by Deanship for Scientific Research at Imam Abdulrahman Bin Faisal University [Project number 2016-575-Med]. The role of the funding organization was purely financial and did not interfere with the study or the publication process.

\section{Ethical Approval}

The ethical approval for the study was obtained from the Institutional Review Board at Imam Abdulrahman Bin Faisal University (number IRB-UGS- 2017-01-092).

\section{Consent}

A written consent was obtained from all participants in the study.

\section{Availability of data and material}

All data are available from the corresponding author upon request.

\section{Authors' contributions}

All authors contributed to the study conception and design. Material preparation was performed by [Khaled R. Alkharsah], sample and data collection were performed by [Ghada Faisal Sharifi], [Alaa Nabil Turkistani], and [Maha Abdel Hadi], data analysis was performed by [Khaled R. Alkharsah], [Haitham Kussaibi], [Ahmed Alsayyah]. The first draft of the manuscript was written by [Khaled R. Alkharsah] and [Ghada Faisal Sharifi]. All authors commented on previous versions of the manuscript. All authors read and approved the final manuscript.

\section{Conflict of interest statement}

The authors declare no conflict of interest

\section{References}

Aguirre-Ghiso JA, Estrada Y, Liu D, et al (2003). ERK(MAPK) activity as a determinant of tumor growth and dormancy; regulation by p38(SAPK). Cancer Res, 63, 1684-95.

Al Balawi IA, Mir R, Abu-Duhier FM (2018). Potential impact of vascular endothelial growth factor gene variation $(-2578 \mathrm{C}>\mathrm{A})$ on breast cancer susceptibility in Saudi Arabia: a Case-Control Study. Asian Pac J Cancer Prev, 19, 1135-43.

Al-Qasem A, Toulimat M, Tulbah A, et al (2012). The p53 codon 72 polymorphism is associated with risk and early onset of breast cancer among Saudi women. Oncol Lett, 3, 875-8.

Al-Qasem AJ, Toulimat M, Eldali AM, et al (2011). TP53 genetic alterations in Arab breast cancer patients: Novel mutations, Asian Pacific Journal of Cancer Prevention, Vol 212167 
pattern and distribution. Oncol Lett, 2, 363-9.

Albalawi IA, Mir R, Abu-Duhier FM (2020). Molecular evaluation of PROGINS mutation in progesterone receptor gene and determination of its frequency, distribution pattern and association with breast cancer susceptibility in Saudi Arabia. Endocr Metab Immune Disord Drug Targets, 20, 760-70.

Bray F, Ferlay J, Soerjomataram I, et al (2018). Global cancer statistics 2018: GLOBOCAN estimates of incidence and mortality worldwide for 36 cancers in 185 countries. $C A$ Cancer J Clin, 68, 394-424.

Casalvieri KA, Matheson CJ, Backos DS, et al (2017). Selective targeting of RSK isoforms in cancer. Trends Cancer, $\mathbf{3}$, 302-12.

DeGraffenried LA, Fulcher L, Friedrichs WE, et al (2004). Reduced PTEN expression in breast cancer cells confers susceptibility to inhibitors of the PI3 kinase/Akt pathway. Ann Oncol, 15, 1510-6.

Easton DF, Pooley KA, Dunning AM, et al (2007). Genome-wide association study identifies novel breast cancer susceptibility loci. Nature, 447, 1087-93.

Eccles SA, Aboagye EO, Ali S, et al (2013). Critical research gaps and translational priorities for the successful prevention and treatment of breast cancer. Breast Cancer Res, 15, R92.

El Bcheraoui C, Basulaiman M, Wilson S, et al (2015). Breast cancer screening in Saudi Arabia: free but almost no takers. PLoS One, 10, e0119051.

Giltnane JM, Balko JM (2014). Rationale for targeting the Ras/ MAPK pathway in triple-negative breast cancer. Discov Med, 17, 275-83.

Johnson N, Speirs V, Curtin NJ, et al (2008). A comparative study of genome-wide SNP, CGH microarray and protein expression analysis to explore genotypic and phenotypic mechanisms of acquired antiestrogen resistance in breast cancer. Breast Cancer Res Treat, 111, 55-63.

Lee YH, Kim JH, Song GG (2014). Genome-wide pathway analysis of breast cancer. Tumour Biol, 35, 7699-705.

Li D, Fu TM, Nan J, et al (2012). Structural basis for the autoinhibition of the C-terminal kinase domain of human RSK1. Acta Crystallogr D Biol Crystallogr, 68, 680-5.

Luu NT, Glen KE, Egginton S, et al (2013). Integrin-substrate interactions underlying shear-induced inhibition of the inflammatory response of endothelial cells. Thromb Haemost, 109, 298-308.

Memish ZA, El Bcheraoui C, Tuffaha M, et al (2014). Obesity and associated factors--Kingdom of Saudi Arabia, 2013. Prev Chronic Dis, 11, E174.

Merdad A, Gari MA, Hussein S, et al (2015). Characterization of familial breast cancer in Saudi Arabia. BMC Genomics, 16, 3.

Michailidou K, Hall P, Gonzalez-Neira A, et al (2013). Largescale genotyping identifies 41 new loci associated with breast cancer risk. Nat Genet, 45, 353-61, 61e1-2.

Mir R, Al Balawi IA, Duhier FMA (2018a). Involvement of microRNA-423 gene variability in breast cancer progression in Saudi Arabia. Asian Pac J Cancer Prev, 19, 2581-9.

Mir R, Javid J, Al Balawi IA, et al (2018b). A germline mutation in the BRCA1 3'UTR variant predicts susceptibility to breast cancer in a Saudi Arabian population. Asian Pac J Cancer Prev, 19, 859-66.

Stacey SN, Manolescu A, Sulem P, et al (2007). Common variants on chromosomes $2 \mathrm{q} 35$ and $16 \mathrm{q} 12$ confer susceptibility to estrogen receptor-positive breast cancer. Nat Genet, 39, 865-9.

Turnbull C, Ahmed S, Morrison J, et al (2010). Genome-wide association study identifies five new breast cancer susceptibility loci. Nat Genet, 42, 504-7.

Xu J, Zhang Y, Berry PA, et al (2011). Growth hormone signaling in human T47D breast cancer cells: potential role for a growth hormone receptor-prolactin receptor complex. $\mathrm{Mol}$ Endocrinol, 25, 597-610.

\section{(ब) $(0 \otimes$}

This work is licensed under a Creative Commons AttributionNon Commercial 4.0 International License. 